GILLES KIROUAC

Coordonnateur Scientifique

Cognition
et Émotions 
(Página deixada propositadamente em branco) 


\title{
GILLES KIROUAC
}

Coordonnateur Scientifique

\section{Cognition et Émotions}

\author{
AUTEURS \\ Klaus R. Scherer \& Janique Sangsue \\ Pierre Philippot \\ Dario Galati \\ Bernard Rimé \\ Pierre Gosselin \\ Ursula Hess \\ Arvid Kappas \& Jean Descôteaux \\ Pio E. Ricci Bitti \\ Pedro Luzes \\ Lise Fillion \\ Ramon Bayés \\ Coimbra - Imprensa da Universidade


COORDENAÇÃO EDITORIAL

Imprensa da Universidade de Coimbra

CONCEPÇÃO GRÁFICA

António Barros

PAGINAÇÃo

António Resende

[Universidade de Coimbra]

EXECUÇÃO GRÁFICA

G.C. - Gráfica de Coimbra, Lda.

Palheira - Assafarge - Apart. 3068

300I-453 Coimbra Codex

ISBN 972-8704-19-4

ISBN 2-7637-81।8-7

DEPÓSITO LEGAL 210430/04

(C) MAIO 2004, Imprensa DA UnIVERSIDAdE DE COIMBRA

\author{
OBRA PUBLICADA COM O PATROCINIO DO \\ INSTITUTO DE PSICOLOGIA COGNITIVA \\ FCT: FUNDAÇÃO PARA A CIÊNCIA E A TECNOLOGIA \\ MINISTÉRIO DA CIÊNCIA E DO ENSINO SUPERIOR \\ apolo do Programa Operacional CiênCIa, TeCnologia, Inovação \\ DO QUADRO COMUNITÁRIO DE APOIO
}




\section{Ramon Bayés}

Universitat Autònoma de Barcelona

\section{PERCEPTION DES SYMPTÔMES ET RÉACTIONS ÉMOTIONNELLES LORS DES MALADIES MORTELLES}

L'émotion, la peur, la souffrance et la douleur sont des mots familiers aussi bien dans le langage de tous les jours que dans la pratique clinique et la recherche dans le domaine des soins palliatifs lors des maladies mortelles.

Les émotions négatives, telle la peur, caractérisent la plupart des mammifères et elles ont évolué pour favoriser l'adaptation et, en général, améliorer la survie des espèces (Darwin, 1872; Izard, 1993; MacLean, 1990; Myers, 1992). La peur est souvent une réaction adaptative. La peur prépare nos corps pour fuir du péril; la peur d'une lésion nous protège du dommage; la peur des représailles contrôle notre agressivité. On croit, en conséquence, que les émotions négatives associées à la douleur et aux pertes sont investies d'une mission biologique et, bien que n'étant apparemment d'aucune utilité pour les malades en fin de vie, elles peuvent, en beaucoup d'autres circonstances, agir comme une ressource efficace pour la personne.

L'idée de souffrance, d'autre part, bien qu'elle ait une signification émotionnelle très claire et de grande importance dans la vie humaine (Cassell, 1982), est très difficile à définir avec précision. Pour Chapman et Gravin (1994) le sentiment d'impuissance est un facteur déterminant dans la souffrance et, après une analyse de la littérature, ces auteurs nous indiquent qu'une définition adéquate de la souffrance doit posséder les éléments suivants: $a$ ) un sentiment de menace à l'intégrité du soi (physique et/ou psychosocial); b) un sentiment d'impuissance et de privation; et c) un caractère émotionnel durable et désagréable. En synthèse, ces auteurs définissent la souffrance comme «un état affectif, cognitif et négatif complexe caractérisé par la sensation d'être menacé dans son intégrité, par le sentiment d'impuissance ressenti face à cette menace et par l'épuisement des ressources 
personnelles et psychosociales qui permettent de l'affronter» (pp. 38). Ses racines se trouvent, probablement, dans la capacité des êtres humains d'anticiper les conséquences des événements (Craig, 1984), laquelle apparaît très tôt puisque Levy (1960) a déjà observé que les enfants, à partir de 6 mois, montrent des réactions de peur sévères devant les médecins et infirmières s'ils ont une histoire de piqûres et de vaccins.

L'International Association for the Study of Pain (IASP), pour sa part, définit la douleur comme étant «...une expérience sensorielle et émotionnelle désagréable, associée à des lésions tissulaires réelles ou probables, ou décrite en fonction de tels dommages» (Cfr. Merskey, 1979). Comme soulignent Chapman et Gavrin (1994), la définition de I'IASP suggère au moins deux types de processus: a) sensoriel, qui livre au cerveau des informations de type spatial, temporel et qualitatif; et b) émotionnel, qui colore de façon négative cette perception sensorielle et qui s'apparente à une menace. En conséquence, l'expérience de la douleur est, partiellement, une expérience émotionnelle et les aspects émotionnels de la douleur sont apparemment discriminables de ses qualités sensorielles (Craig, 1984).

Dans une recherche déjà classique, Beecher (1956, 1959) a montré dramatiquement l'importance des facteurs psychologiques dans la modulation de la douleur en comparant les données précédentes de 150 soldats des États Unis qui ont été blessés sur la plage d'Anzio pendant la deuxième guerre mondiale, avec celles de 150 patients civils du même âge habitant les États Unis et qui ont été soumis à une intervention chirurgicale avec une affection similaire de leur organisme. Tandis que seulement 32\% des premiers ont manifesté qu'ils avaient besoin d'analgésiques pour combattre la douleur, $83 \%$ des civils réclamaient ce type de médicaments. Pour Beecher, «il n'existe pas une relation simple entre la blessure per se et la douleur. La douleur se trouve déterminée, en bonne partie, par d'autres facteurs et, dans ce cas-là, la signification des 212 blessures pour les affectés était de la plus grande importance». Pour les soldats, les blessures sont associées au retour au foyer et à la fin des combats; pour les civils, les blessures n'ont que des connotations négatives et peuvent être considérées comme une menace contre l'intégrité physique. Comme le souligne Craig (1984), «la perception de la douleur peut être atténuée ou accentuée dans les processus émotionnels» (pp. 155). La peur, l'anxiété et la dépression peuvent amplifier la douleur et les thérapeutiques pharmacologiques et psychologiques qui diminuent l'anxiété et la tension réduisent souvent la douleur clinique. 
Baines (1990), conseillère médicale du St. Christopher Hospice de Londres, en référence à la douleur des malades en fin de vie nous signale: «C'est probablement assez exceptionnel que la douleur soit produite par des facteurs non physiques. Néanmoins, les problèmes émotionnels et sociaux rendent souvent plus violente la douleur et, de fait, il s'établit un cercle vicieux dans lequel la douleur physique conduit vers l'anxiété et la dépression, et ces dernières, à leur tour, diminuent le seuil des sensations douloureuses»). Mount (1984), l'introducteur des unités de soins palliatifs au Canada, écrit que «la douleur du cancer a son origine dans une pathologie physique nuisible, mais elle est toujours modifiée par l'esprity (pp. 460). Cassell (1982) nous montre que la signification perçue de la douleur influe dans la quantité d'analgésique qui sera nécessaire pour la contrôler. Et Stedeford (1984), finalement, nous signale que quand les symptômes comme la douleur n'apportent aucun soulagement avec les mesures thérapeutiques qui sont habituellement effectives, le médecin doit soupçonner que ce sont des problèmes psychologiques qui les rendent plus violents ou encore qui les provoquent. Clairement, les processus psychologiques ne peuvent être ignorés même dans les cas où il existe une base biologique solide pour la douleur.

La douleur et la souffrance se partagent la dimension négative de l'émotion, mais la relation entre elles est imprécise. On peut bien éprouver de la douleur et ne pas souffrir, c'est à dire, bien que la douleur puisse être désagréable, elle ne cause pas de souffrance si la personne la perçoit comme non menaçante - par exemple, dans l'accouchement sans problèmes ou dans le rapatriement des soldats blessés de Beecher $(1956,1959)$ - et, à l'inverse, on peut aussi souffrir et ne pas éprouver de la douleur - comme dans l'attente du diagnostic d'une biopsie ou d'une preuve sérologique au VIH, occasions dans lesquelles la personne éprouve, dans l'impuissance, un sentiment de menace. Donc, il ne suffit pas d'éprouver des douleurs pour souffrir et on peut souffrir sans éprouver une douleur physique. Cependant, il y a de fortes chances que la douleur - surtout en cas de maladie grave - provoque de la souffrance. Ce n'est pas que toute souffrance ait son origine dans la douleur - comme l'ont écrit Loeser et Melzack (1999) mais dans notre culture médicalisée, nous exprimons la souffrance dans le langage de la douleur.

Ce que nous disons de la douleur nous pouvons l'étendre, chez un grand malade, à d'autres symptômes physiques (Bruera, 1993) perçus par lui comme menaçants, comme, par exemple, la dyapnée, l'insomnie, la faiblesse, 
la nausée, la diarrhée ou le vomissement. Et, de façon pareille à la douleur, on peut bien éprouver de la dyapnée et de la faiblesse, par exemple, après une course athlétique, ou de l'insomnie si on remporte le grand prix du loto, et ne pas souffrir pour eux.

Non seulement des appréhensions d'ordre physique assaillent le malade en fin de vie - peur de la douleur, de perdre son image corporelle, son autonomie, peur des complications qui peuvent survenir, peur de l'agonie mais bien d'autres peurs sont souvent présentes: peur d'être dépendant des autres, de perdre le contrôle de la situation, peur de ne pas avoir le temps d'accomplir des souhaits intimes, peur de la séparation, de l'avenir pour ceux qui resteront, de l'incertitude que «l'après mort» pose aux croyants.

Il faut aussi penser que ces peurs agissent sur une personne dont l'esprit peut être calme, mais qui peut être aussi dépressif ou anxieux.

Nous pouvons représenter ces idées précédentes de la façon que montre le modèle de la Figure I (Bayés, Arranz, Barbero y Barreto, 1996).

Perception

sensorielle

désagréable

(douleur,

dyapnée, etc.)

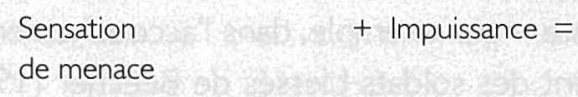

\section{Souffrance}

État d'esprit

Perception

psychosociale

désagréable

(séparation,

perte de contrôle,

etc.)

(Bayés, Arranz, Barbero et Barreto, 1996)

Figure I - Relation entre perception sensorielle et/ou psychosociale désagréable, et souffrance 
À mesure qu'une maladie grave - le cancer, le SIDA, etc. - avance, les réactions psychologiques opèrent une intégration complexe entre la mémoire des expériences passées, la perception des menaces futures et les ressources disponibles (Razavi et Delvaux, 1994). La phase terminale est souvent riche d'événements et d'échanges vécus intensément. Des pertes de rôles et de fonctions peuvent constituer des crises existentielles.

En résumé, nous avons peur:

a) de ne pouvoir jamais récupérer ce que nous avons déjà perdu.

b) de perdre tout ce que nous avons encore.

c) de ne pouvoir avoir jamais ce qui nous manque et que nous souhaitons intensivement d'avoir.

Les symptômes physiques, les réactions d'autrui et les situations perçues par le malade comme menaçantes sont les signes qui déclenchent la peur et la souffrance. Alors, il sera très important, si nous voulons aider ce malade, de connaître quelles sont, personnellement, pour lui et dans chaque moment précis, les choses qui l'inquiètent et qui, peut être, rendent sa vie un enfer.

En effet, d'une part, il y a des peurs, on peut dire biologiques, qui ont besoin, pour faire leur apparition dans des individus d'une espèce précise, d'un apprentissage faible ou presque nul, comme la peur des serpents et des araignées, et surtout, la peur de la mort. Lorsque Donald Hebb (1980) a montré pour première fois un modèle en argile de tête de chimpanzé à un groupe d'animaux, ils ont été pris de panique, et leur réaction a été semblable à celle des personnes qui voient le corps mort ou écartelé d'un être humain. Mais, d'autre part, le long de notre histoire personnelle dans une culture précise, moyennant de complexes associations, des observations et des interactions, nous pouvons apprendre à craindre presque quoi que ce soit et à avoir peur de choses ou d'événements différents de ceux qui font peur à d'autres. Le seul moyen de connaître quels symptômes, comportements et situations ont atteint la condition de menaçants pour une personne est de chercher à connaître quelles sont les choses où les situations qui l'inquiètent.

Pour la plupart des personnes concernées, la mort induit un vécu de rupture et de séparation définitives. Toutes ces réactions peuvent mener à une adaptation ou à un échec (Lipowski, 197I; Lazarus, 1982) et souvent peuvent donner lieu à une détresse émotionnelle qui se traduit en termes d'anxiété et de dépression. 
Si nous partons de la définition de Chapman et Gravin (1994), il est évident qu'on peut aborder la souffrance du malade de différentes façons, complémentaires et non alternatives:

a) Avec le contrôle de la douleur et d'autres symptômes perçus par le patient comme menaçants - une douleur sévère peut suffire pour causer et maintenir la souffrance - et pour que les soignants puissent agir efficacement, il faudra se demander souvent quels sont ces symptômes biologiques, psychologiques ou sociaux - perçus comme menaçants. De fait, il faut construire et soutenir dès le début une relation de confiance entre les soignants, le patient et sa famille. Quand la maladie est terminale, l'assurance que les soignants ont l'intention de contrôler les symptômes perçus comme menaçants contribue pour beaucoup à prévenir la souffrance. II faut se souvenir, par exemple, que toute difficulté respiratoire peut s'accompagner d'anxiété chez le malade et ses familiers (Mouren-Mathieu, 1987). Elle trouble le sommeil du malade autant pour des raisons physiques que par crainte de ne pas se réveiller. Pour répondre efficacement à cette angoisse, il faut connaître les peurs du malade: le manque d'air?, l'ignorance de la cause de ses difficultés?, la mort?, des préoccupations familiales?. Pour Mouren-Mathieu (1987), dans cette situation, il faut rassurer le malade en l'informant sur l'étiologie de sa dyapnée en lui posant des questions telles comme «Avez-vous peur de quelque chose?»». Personnellement, nous préférons la question «Avez-vous du souci pour quelque chose?».

b) Avec la minimalisation de l'impuissance qu'il ressent, en l'aidant à réajuster ses buts sur une base quotidienne. II faut encourager le malade à accepter ses limitations et se poser des objectifs réalistes: lui faire accepter progressivement une canne, une chaise roulante qui lui conserveront une autonomie relative: pouvoir encore sortir ou se mettre à table.

c) En lui maximisant ses propres ressources et en lui en fournissant de nouvelles - comme, par exemple, la relaxation - pour contrôler le mieux possible ses émotions et ses activités cognitives, alors que ses capacités physiques déclinent abruptement. En aidant le patient à acquérir le sentiment de «maîtriser la morts, le soignant contribue à prévenir la souffrance du patient et de sa famille.

Quant au problème de l'information, il faut souligner, avec Gómez Sancho (1994), que «La vérité est l'antidote de la peur. Les événements terribles et connus sont préférables aux événements terribles mais inconnus» (pp. 287).

Pour accomplir le programme d'interventions précédent, il est nécessaire que nous puissions connaître: 
a) Quels symptômes physiques sont pour le malade une source de menace

b) Quels facteurs psychosociaux sont pour le malade une source de menace.

c) Quelle information possède la personne sur sa maladie, son traitement et sa situation.

d) De quels recours dispose le malade pour affronter la situation.

La présence imminente de la mort - sa propre mort ou celle d'une personne bien-aimée - est, à notre avis, une situation naturelle de choix pour l'étude des émotions plus intenses que peut éprouver l'homme. Vachon (1993) nous signale que les données correspondant à des échantillons représentatifs randomisés de sous populations du Canada nous montrent qu'entre le 61 et le 79 pour cent des patients des unités de soins palliatifs présentent une douleur psychologique (distress) sévère par rapport à 18 -34 pour cent chez le groupe de comparaison.

De plus, son intérêt comme sujet de recherche est, à notre avis, prioritaire pour les professionnels de la santé, étant donné que plus nous pourrons connaître et maîtriser la souffrance plus nous pourrons aider un grand nombre de personnes à parcourir, sans peur - ou avec la peur diminuée ou contrôlée - et avec dignité, le dernier chapitre de leur existence. La connaissance et le contrôle de la souffrance représentent pour nous tous, chercheurs et cliniciens, un formidable défi.

Et si nous voulons arriver au bout, si nous voulons construire des équipes multidisciplinaires de soins palliatifs efficaces (Bayés et Barreto, 1992), nous devons, avant tout, connaître quels sont, de façon évolutive vers la mort, les peurs, les soucis, les symptômes - biologiques, psychologiques, sociaux - perçus comme menaçants par les patients en phase terminale.

C'est dans ce but qu'il y a quelques années nous avons pris part à une recherche menée dans deux hôpitaux espagnols qui ont des services de soins palliatifs (Comas, Martínez, Barreto, Bayés, Roca, Pascual, Gómez-Batiste, y García Conde, 1993) et que, lorsque cette recherche a été finie, nous nous sommes engagés dans un projet multicentre avec le concours de quinze hôpitaux (Bayés, Barreto, Comas y Limonero, 1997).

Les données que je vous présente maintenant correspondent à 37। malades ( 314 de cancer et 57 de SIDA) en fin de vie avec un traitement thérapeutique exclusivement palliatif. 
Le même questionnaire - avec 33 symptômes issus de la littérature et des résultats d'une recherche précédente (Comas et al., 1993) a été administré à chaque malade jusqu'au moment de sa mort, de façon systématique, le plus grand nombre de fois qu'il a été possible, en accord avec le principe éthique de profit du malade et d'opportunité clinique. Le but principal de cette recherche prospective a été de connaître l'évolution de la perception des symptômes et des soucis à mesure que la mort approche (Cfr. Bayés, 1995).

II faut se souvenir qu'une caractéristique des malades atteints du cancer et du SIDA en fin de vie - en contraste avec les malades chroniques - est le changement fréquent de leurs symptômes et problèmes (Sanz, Gómez-Batiste, Gómez-Sancho et Núñez Olarte, 1993; Chochinov, Tataryn, Clinch y Dudgeon, 1999).

Dans le Tableau I vous pouvez trouver les 15 symptômes perçus par le plus grand nombre de malades (Bayés y Limonero, 1999)

TABLEAU I - Symptômes les plus perçus par les malades en fin de vie

\begin{tabular}{|c|c|c|}
\hline & Synptôme & $\begin{array}{c}\% \text { de malades qui } \\
\text { le perçoivent }\end{array}$ \\
\hline 1) & Faiblesse générale & 86 \\
\hline 2) & Perte de poids & 72 \\
\hline 3) & Somnolence diurne & 68 \\
\hline 4) & Tristesse & 65 \\
\hline & Anorexie & 65 \\
\hline 5) & Nervosité & 63 \\
\hline 6) & Douleur & 57 \\
\hline 7) & Constipation & 53 \\
\hline 8) & Troubles de sommeil & 52 \\
\hline 9) & Problèmes de vision & 44 \\
\hline 10) & Problèmes de respiration & 43 \\
\hline 11) & Troubles de langage & 41 \\
\hline 12) & Nausée ou vomissement & 39 \\
\hline 13) & Mal à la bouche & 38 \\
\hline 14) & Toux & 37 \\
\hline
\end{tabular}

(Bayés et Limonero, 1999) 
De ce Tableau, nous pouvons déjà déduire qu'il n'y a aucun symptôme qui soit perçu par tous les malades. II n'y a pas de symptômes universels. II n'y a pas de maladies, mais des malades.

Dans le Tableau 2, d'autre part, nous pouvons observer, à partir des symptômes qui sont perçus, quels sont ceux qui préoccupent le plus les malades.

TABLEAU 2 - Symptômes perçus qui inquiètent le plus les malades en fin de vie

\begin{tabular}{|c|c|c|}
\hline \multicolumn{2}{|r|}{ Symptôme } & $\begin{array}{l}\text { \% de malades qui s'en inquiètent } \\
\text { par rapport à ceux qui le perçoivent }\end{array}$ \\
\hline 1) & Faiblesse générale & 50 \\
\hline 2) & Anorexie & 45 \\
\hline 3) & Paralysie & 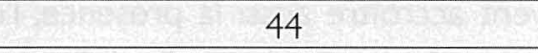 \\
\hline 4) & Douleur & 38 \\
\hline 5) & Perte de poids & 32 \\
\hline 6) & Problèmes de respiration & 30 \\
\hline & Constipation & 30 \\
\hline 7) & Nausée ou vomissement & 27 \\
\hline & Troubles de sommeil & 26 \\
\hline & Tristesse & 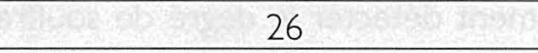 \\
\hline 9) & Aspect physique & 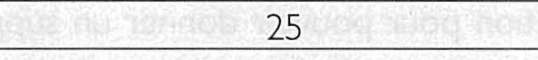 \\
\hline & Mal à la bouche & 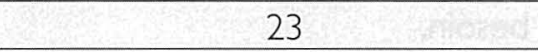 \\
\hline 11) & Solitude & 22 \\
\hline 12) & Somnolence diurne & 20 \\
\hline 13) & Difficultés de déglutition & 19 \\
\hline
\end{tabular}

(Bayés et Limonero, 1999)

De ces deux Tableaux, nous pouvons déduire:

a) Les symptômes perçus par le plus grand nombre de malades ne sont pas nécessairement ceux qui les inquiètent le plus (p.ex.: somnolence diurne, constipation et problèmes visuels). 
b) Quelques-uns des symptômes qui sont perçus par un nombre relativement petit de malades produisent un grand souci (p.ex.: paralysie, problèmes de respiration et mal à la bouche).

c) Quant aux symptômes physiques les plus inquiétants (faiblesse générale, anorexie, paralysie, douleur et problèmes de respiration), entre la moitié et les $2 / 3$ des malades qui les perçoivent ne les considèrent pas très menaçants, ce qui nous fait penser au schéma de la Figure 1.

d) Le degré de menace de chaque symptôme particulier dépendra de l'histoire et des caractéristiques personnelles du malade. Alors, la seule façon de connaître si un des symptômes perçus est pour lui cause de souffrance sera de le questionner, d'une façon éthique, sur le degré d'inquiétude que ce symptôme produit chez lui.

e) Quelques symptômes psychologiques, comme la tristesse (dépression) et le nervosité (anxiété), et d'autres symptômes psychosociaux, comme la solitude ou la dépendance, provoquent un degré de souci considérable et peuvent accroître aussi la présence, l'intensité ou la sensation de menace des symptômes physiques. Ils doivent, donc, si possible, être contrôlés.

De façon évolutive, nous avons observé, par exemple, que la tristesse augmente dans les 30 jours qui précédent le moment de la mort.

Dans le présent contexte, notre intention n'est pas de parler de mesures thérapeutiques particulières. Mais elle est, peut être, de nous questionner comment détecter le degré de souffrance de chaque malade dans chaque situation pour pouvoir donner un support prioritaire a ceux qui en ont le plus besoin.

Dans ce but, dans un contexte clinique, notre modèle de criblage (screening) est le thermomètre: II est facile à appliquer; on peut le faire servir autant de fois qu'on le désire; et il nous indique si les choses vont mieux, si elles vont plus mal ou si elles se maintiennent sans variation. Évidemment, comme la souffrance est subjective, il faudra questionner le malade et nos questions ne devront pas, pour des raisons éthiques, troubler et augmenter sa souffrance. Est-ce qu'il est possible d'avoir un thermomètre clinique de la souffrance humaine qui accomplisse ces conditions?

Nous croyons que la réponse est affirmative. Notre idée est issue d'un vieux texte de William James (1890) qui a plus de cent ans et dans lequel l'auteur nous parle des différences entre le temps chronologique et le temps subjectif. 
De fait, l'expérience subjective nous montre que, quand nous nous trouvons dans une situation agréable, le temps perçu nous semble plus court que le temps chronologique; mais, si la situation est désagréable, le temps s'allonge et peut se faire interminable. Nous croyons que questionner un malade sur sa perception subjective d'une période chronologique particulière, peut être un indicateur bon et simple du degré de bien-être ou de malaise que le malade éprouve dans la période considérée (Bayés, Limonero, Barreto y Comas, 1995). Évidemment, avec cette méthode, nous ne pouvons savoir si la cause d'un possible malaise est une profonde souffrance ou un simple ennui. Pour le dévoiler, il nous faudra continuer l'exploration en profondeur (Bayés, 2000). Mais nous nous trouvons dans un problème semblable avec le thermomètre clinique. II nous indique la fièvre mais non pas ses causes. Pour les connaître il nous faudra interroger le malade, l'explorer, lui demander des analyses.

Notre thermomètre émotionnel est un simple instrument de criblage. Mais il nous permet, d'une façon simple et éthique, de détecter quelques-uns des malades qui ont le plus besoin de notre aide.

Nous avons étudié avec cette méthode 655 situations différentes chez les 37 I malades en fin de vie de notre recherche. Nos résultats montrent que dans $83 \%$ des cas dans lesquels les malades nous indiquent qu'ils ont perçu le temps comme «Long» ou «Très long» ils qualifient leur état général comme «Médiocre», «Mauvais» ou "Très mauvais» et que dans le 76,8\% des cas où ils nous indiquent qu'ils ont expérimenté le temps comme "Court» ou «Très court» leur degré de bien-être a été perçu comme «Bon» ou «Très bon». Ces données sont statistiquement significatives $\left(X^{2}=129,7\right.$, d.I. 2, $\mathrm{P}<0.00$ I) (Bayés, Limonero, Barreto et Comas, 1997).

L'étude des émotions et des cognitions devant la mort est un sujet important et difficile. Ces émotions et cognitions constituent les éléments d'une situation que nous devrons tous vivre un jour. Mieux les connaître nous aidera, peut-être, à l'affronter avec moins de peur et de souffrance, et à aider d'autres êtres humains à faire de même.

\section{RÉFÉRENCES}

Baines, M. (1990). Tackling total pain. En C. Saunders (Ed.) Hospice and palliative care (pp. 26-38). Londres: Arnold.

Bayés, R. (1995). SIDA y psicología. Barcelona: Martínez Roca. 
Bayés, R. (2000). Una estrategia para la detección del sufrimiento en la práctica clínica. Revista de la Sociedad Española del Dolor, 7, 70-74.

Bayés, R., Arranz, P., Barbero, J. et Barreto, P. (1996). Propuesta de un modelo integral para una intervención terapéutica paliativa. Medicina Paliativa, 3, 114-121.

Bayés, R. et Barreto, P. (1992). Las unidades de cuidados paliativos como ejemplo de interdisciplinariedad. Clínica y Salud, 3 (1), I I-19.

Bayés, R., Barreto, P., Comas, M.D. et Limonero, J.T. (1997). Multicentric research on assessment of symptoms and worries perceived by terminally ill patients. Dans F. De Conno (Ed.) Proceedings of the IV Congress of the European Association of Palliative Care (pp. 579-582). Milan: European Association of Palliative Care.

Bayés, R. et Limonero, J.T. (1999). Prioridad en el tratamiento de los síntomas que padecen los enfermos oncológicos en situación terminal. Medicina Paliativa, 6, 19-21.

Bayés, R., Limonero, J.T., Barreto, P. et Comas, M.D (1995). Assessing suffering. The Lancet, 346, 1492.

Bayés, R., Limonero, J.T., Barreto, P. et Comas, M.D. (1997). A way to screen for suffering in palliative care. Journal of Palliative Care, 13 (2), 22-26.

Beecher, H.K. (1956). Relationship of significance of wound to the pain experience. Journal of The American Medical Association, 161, 1609-1613.

Beecher, H.K. (1959). Measurement of subjective responses: quantitative effects of drugs. Nueva York: Oxford University Press.

Bruera, E. (1993). Research in symtoms other than pain. Dans D. Doyle, G.W.C. Hanks et N. MacDonald (Eds.) Oxford texbook of palliative care (pp. 87-92). Oxford: Oxford University Press.

Cassell, E.J. (1982). The nature of suffering and the goals of Medicine. The New England Journal of, 306, 639-645.

Comas. M.D., Martínez, E, Barreto, M.P., Bayés, R., Roca, J., Pascual, A., Gómez-Batiste, X. et García Conde, J. (1993). Assessment of the perception of symptoms and worries in Spanish terminal patients (IV). Comunication presenté au 3er Congreso Latini Dies. Toulouse, Mai.

Craig, K.D. (1984). Emotional aspects of pain. Dans P.D. Wall et R. Melzack (Eds.) Texbook of pain (pp. 153- 161). Edinburg: Churchill Livingstone.

Chapman, C.R. et Gavrin, J. (1994). La souffrance et sa relation avec la douleur. Infokara, $n^{\circ} 34,24-43$.

Chochinov, H.M., Tataryn, D., Clinch, J.J. et Dudgeon, D. (1999). Will to life in the terminally ill. The Lancet, 354, 816-819.

Darwin, C. (1872). The expression of emotions in man and animals. London: John Murray.

222 Gómez Sancho, M. (Ed.) (1994). Cuidados paliativos e intervención psicosocial en enfermos terminales. Las Palmas de Gran Canaria: Instituto Canario de Estudios y Promoción Social y Sanitaria.

Hebb, D.O. (1980). Essay on mind. Hillsdale, N.J:: Erlbaum.

Izard, C. (1993). Four systems for emotion activation: Cognitive and noncognitive processes. Psychological Review, 100, 68-90.

James, W. (1890). Principles of psychology (Edition de 1980). New York: Holt

Lazarus, R.S. (1982). Stress and coping as factors in health and illness. Dans J. Cohen et al. (Eds.) Psychosocial aspects of cancer (pp. 163-190). New York: Raven.

Levy, D.M. (1960). The infant's earliest memory of inoculation. Journal of Genetic Psychology, $96,3-46$. 
Lipowski, Z. (1971). L'individu face à la maladie psychique. Revue de Médecine Psychosomatique et de Psychologie Médicale, 13 (3), 235-249.

Loeser, J.D. et Melzack, R. (1999). Pain: an overview. The Lancet, 353, 1607-1609.

MacLean, P.D. (1990). The triune brain in evolution: Role in paleocerebral functions. New York: Plenum.

Merskey, H. (1979). Pain terms: A list with definitions and a note on usage. Recommended by the International Association for the Study of Pain (IASP) Subcommitttee on Taxonomy. Pain, 6, 249-252.

Mount, B.M. (1984). Psychological and social aspects of cancer pain. Dans P.D. Wall et R. Melzack (Eds.) Texbook of pain (pp. 460- 47I). Edinburg: Churchill Livingstone.

Mouren-Mathieu, A.M. (1987). Soins palliatifs. Montréal: Les Presses de l'Université de Montréal.

Myers, D. (1992). Psychology (3e ed.). Worth. Traduction espagnole: Psicología. Madrid: Panamericana

Razavi, D. et Delvaux, N. (1994). Psycho-oncologie Paris: Masson.

Sanz, J., Gómez-Batiste, X., Gómez Sancho, M. et Núñez Olarte, J.M. (1993). Cuidados paliativos: Recomendaciones de la Sociedad Española de Cuidados Paliativos (SECPAL). Madrid: Ministerio de Sanidad y Consumo.

Stedeford, A. (1984). Facing death: patients, families and professionals. Oxford: Heinemann.

Vachon, M.L.S. (1993). Emotional problems in palliative medicine: patient, family, and professional. Dans D. Doyle, G.W.C. Hanks et N. MacDonals (Eds.) Oxford textbook of palliative medicine (pp. 577-605). Oxford: Oxford University Press. 
Série

Investigaçāo

Coimbra

Imprensa da Universidade

Les Presses de L'Université Laval

Les Editions de L'IORC

2004 